Article

\title{
The Influence of the Framework and Extraframework Content on the High Pressure Behavior of the GIS Type Zeolites: The Case of Amicite
}

\author{
Rossella Arletti ${ }^{1,2}$, Carlotta Giacobbe ${ }^{3}$, Simona Quartieri ${ }^{4}$ and Giovanna Vezzalini ${ }^{5, *}$ \\ 1 Dipartimento di Scienze della Terra, Università di Torino, Via Valperga Caluso 35, 10125 Torino, Italy; \\ rossella.arletti@unito.it \\ 2 CrisDI Interdipartemental Center, University of Torino, Via Giuria 7, 10125 Torino, Italy \\ 3 ESRF-European Synchrotron Radiation Facility, CS 40220-38043 Grenoble Cedex 9, Grenoble, France; \\ giacobbe@esrf.fr \\ 4 Dipartimento di Scienze Matematiche e Informatiche, Scienze Fisiche e Scienze della Terra, \\ Università di Messina, Viale Ferdinando Stagno d'Alcontres 31, 98166 Messina S. Agata, Italy; \\ squartieri@unime.it \\ 5 Dipartimento di Scienze Chimiche e Geologiche, Università di Modena e Reggio Emilia, Via G. Campi 103, \\ 41125 Modena, Italy \\ * Correspondence: mariagiovanna.vezzalini@unimore.it; Tel.: +39-059-205-8471
}

Academic Editor: Annalisa Martucci

Received: 30 November 2016; Accepted: 29 January 2017; Published: 5 February 2017

\begin{abstract}
This paper reports a study, performed by in-situ synchrotron X-ray Powder Diffraction, of the high pressure behavior of the natural zeolite amicite $\left[\mathrm{K}_{4} \mathrm{Na}_{4}\left(\mathrm{Al}_{8} \mathrm{Si}_{8} \mathrm{O}_{32}\right) \cdot 10 \mathrm{H}_{2} \mathrm{O}\right]$, the GIS-type phase with ordered $(\mathrm{Si}, \mathrm{Al})$ and $(\mathrm{Na}, \mathrm{K})$ distribution. The experiments were carried out up to 8.13(5) GPa in methanol:ethanol:water = 16:3:1 (m.e.w.) and 8.68(5) GPa in silicone oil (s.o.). The crystal structure refinements of the patterns collected in m.e.w. were performed up to 4.71(5) GPa, while for the patterns collected in s.o. only the unit cell parameters were determined as a function of pressure. The observed framework deformation mechanism-similar to that reported for the other studied phases with GIS topology-is essentially driven by the distortion of the "double crankshaft" chains and the consequent changed shape of the 8-ring channels. The pressure-induced over-hydration observed in the experiment performed in aqueous medium occurs without unit cell volume expansion, and is substantially reversible. A comparison is made with the high pressure behavior of the other GIS-type phases, and the strong influence on compressibility of the chemical composition of both framework and extraframework species is discussed.
\end{abstract}

Keywords: zeolite; amicite; high pressure; compressibility; in-situ synchrotron XRPD; pressure-induced hydration (PIH); structure refinement

\section{Introduction}

In the last 15-20 years, studies on the behavior of both natural and synthetic microporous materials under high pressure (HP) have multiplied noticeably, providing not only important information on their elastic behavior and stability, but also opening new perspectives for technological applications. For instance, among the physical properties of microporous materials investigated under compression, worthy of mention are: the so called P-induced amorphization processes (PIA) (e.g., [1-6]), the effect of pressure on the ionic conductivity (e.g., [7,8]), the P-induced over-hydration (PIH) (e.g., [9-14]) and the penetration of gas, like $\mathrm{Ar}, \mathrm{Xe}$, and $\mathrm{CO}_{2}$ [15-17]. High pressure experiments on porous materials have recently led to the synthesis of linear carbon based polymers in pure silica zeolites. Linear polymers like polyacetylene (PA), polyethylene (PE), and polycarbonyl ( $\mathrm{pCO}$ ) have been obtained 
from compression resulting in nanocomposite organic/inorganic materials, which are good candidates for developing highly directional semiconductors and high energy materials [18].

The HP behavior of zeolites when compressed in non-penetrating fluids has recently been reviewed by Gatta and Lee (2014) [19] and summarized in the following way: (i) microporosity does not necessarily imply high compressibility, in fact the range of compressibility is wide, with bulk modulus $\mathrm{K}_{0}$ ranging from $\sim 15$ to $\sim 70 \mathrm{GPa}$; (ii) the flexibility observed in zeolites is based mainly on tetrahedra tilting; (iii) the deformation mechanisms are dictated by the framework topology; (v) the extraframework content (cations and water molecules) governs the compressibility level in isotypic structures.

Zeolites with GIS topology [20] and GIS-like materials have been studied under both high temperature and high pressure, revealing widely variable degrees and mechanisms of deformation as a function of the non-ambient experimental conditions and the chemical composition of both the framework and extraframework. The study of gismondine dehydration [21] showed that this framework is particularly flexible.

The HP behavior of a natural gismondine was studied using both "non-penetrating" (i.e., silicone oil, s.o.) [22] and "penetrating" (methanol:ethanol:water = 16:3:1, m.e.w.) [11] pressure-transmitting media (PTM). In the latter case, a PIH effect was observed at a very low P, inducing full occupation of originally partially occupied water sites. On the whole, both experiments revealed an unexpected low compressibility of gismondine, notwithstanding the high flexibility showed by this framework during dehydration and the similar framework deformation mechanisms [21].

Lee et al. [23] and Jang et al. [24] studied the HP behavior of two synthetic phases with GIS topology, both compressed in penetrating media: a K-gallosilicate (K-GaSi-GIS) and a $\mathrm{K}$-aluminogermanate (K-AlGe-GIS), respectively. These studies highlighted a very different response to hydrostatic pressure in materials sharing the same GIS topology, but with considerably different framework and extraframework compositions.

Two microporous mixed octahedral-pentahedral-tetrahedral (OPT; [25]) framework silicates, structurally related to the GIS topology, were studied under HP [26]: cavansite and pentagonite, the orthorhombic dimorphs of $\mathrm{Ca}(\mathrm{VO})\left(\mathrm{Si}_{4} \mathrm{O}_{10}\right) \cdot 4 \mathrm{H}_{2} \mathrm{O}$. When compressed in m.e.w., these two phases exhibit rather different behaviors: pentagonite undergoes $\mathrm{PIH}$, thanks to the crucial role of the seven-fold coordinated $\mathrm{Ca}$, suitable for accepting an additional $\mathrm{H}_{2} \mathrm{O}$ molecule. In contrast, in cavansite the eight-fold coordinated Ca cations do not allow further water penetration and thus $\mathrm{PIH}$ is not observed. The higher compressibility in s.o. of cavansite compared to gismondine is attributed to the presence of $\mathrm{VO}_{5}$ pyramids connecting the tetrahedral layers of the vanadosilicate.

This paper presents a study, performed by in-situ synchrotron X-ray Powder Diffraction (XRPD), of the HP stability and behavior of the natural zeolite amicite $\left[\mathrm{K}_{4} \mathrm{Na}_{4}\left(\mathrm{Al}_{8} \mathrm{Si}_{8} \mathrm{O}_{32}\right) \cdot 10 \mathrm{H}_{2} \mathrm{O}\right]$, the GIS phase with ordered $(\mathrm{Si}, \mathrm{Al})$ and $(\mathrm{Na}, \mathrm{K})$ distribution. The investigation aimed in particular to understand: (i) the relationships between compressibility and framework/extraframework content; (ii) the influence of different PTM (penetrating 16:3.1 m.e.w. and non-penetrating s.o., respectively) on the compressibility and HP deformation mechanisms of this zeolite.

\section{Amicite Structure}

Amicite [ideal formula $\mathrm{K}_{4} \mathrm{Na}_{4}\left(\mathrm{Al}_{8} \mathrm{Si}_{8} \mathrm{O}_{32}\right) \cdot 10 \mathrm{H}_{2} \mathrm{O}$ ] is a rare natural zeolite, classified as the ordered $\mathrm{K}, \mathrm{Na}$ member of the gismondine group [27]. The sample used for this study is from the type locality (Höwenegg in Hegau, southern West Germany)—where amicite was discovered associated with merlinoite in a basaltic rock-and is the same studied by Alberti and Vezzalini [28] (chemical formula: $\mathrm{K}_{3.75} \mathrm{Na}_{3.61} \mathrm{Ca}_{0.05}\left[\mathrm{Al}_{7.86} \mathrm{Si}_{8.24} \mathrm{O}_{32}\right] \cdot 9.67 \mathrm{H}_{2} \mathrm{O}$ ). Its GIS framework topology is shared by the other natural zeolites gismondine, garronite, gobbinsite, and by several other synthetic phases. Amicite structure [28] was determined in the monoclinic $I 2$ s.g. The cell parameters are $a=10.226(1)$, $b=10.422(1), c=9.884(1) \AA, \beta=88^{\circ} 19(1)$. The framework can be described as intersecting ribbons of 4-membered rings of tetrahedra (defined as double-crankshaft chains) running in the $a$ and $c$ directions 
(Figures 1 and 2), laterally linked to form two sets of channels delimited by 8-membered rings running parallel to [100] and [001]. The ordered distribution of $\mathrm{Si}$ and $\mathrm{Al}$ in the tetrahedra, and of $\mathrm{Na}$ and $\mathrm{K}$ in the channels, induces a lowering in symmetry from the topological $I 4_{1} / a m d$ space group to the real one I2. Na and $\mathrm{K}$ are distributed in two different and fully occupied sites, with the water molecules in four sites, three of which are fully occupied (W1, W2, W3). Na is coordinated to three framework oxygen atoms and to all the water molecules, while $\mathrm{K}$ is coordinated to four framework oxygen atoms and the three fully occupied water sites.

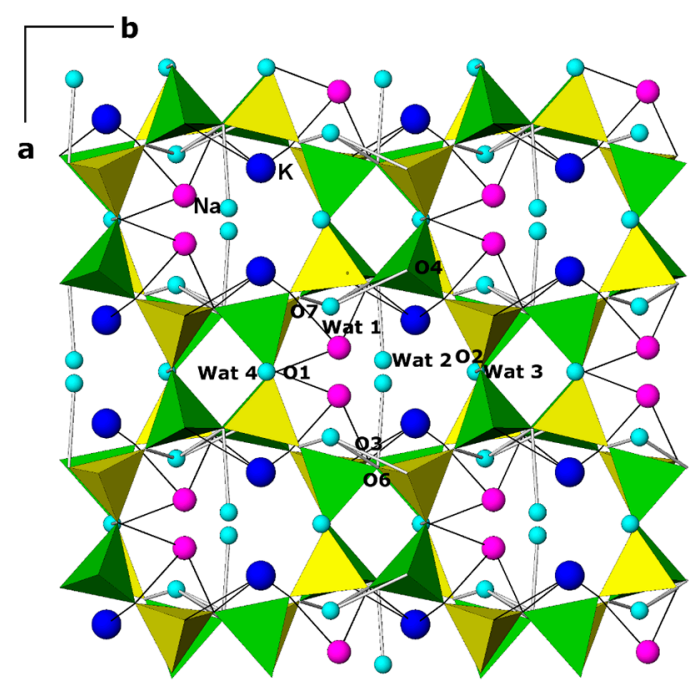

a

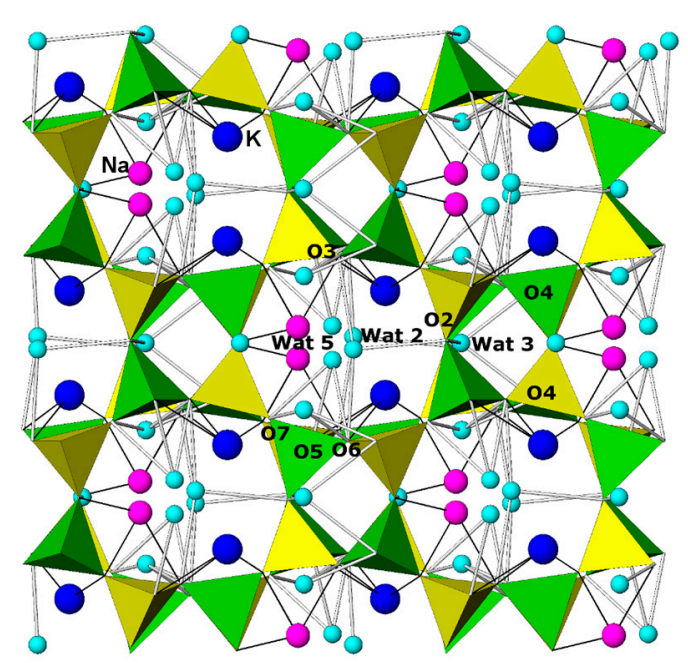

b

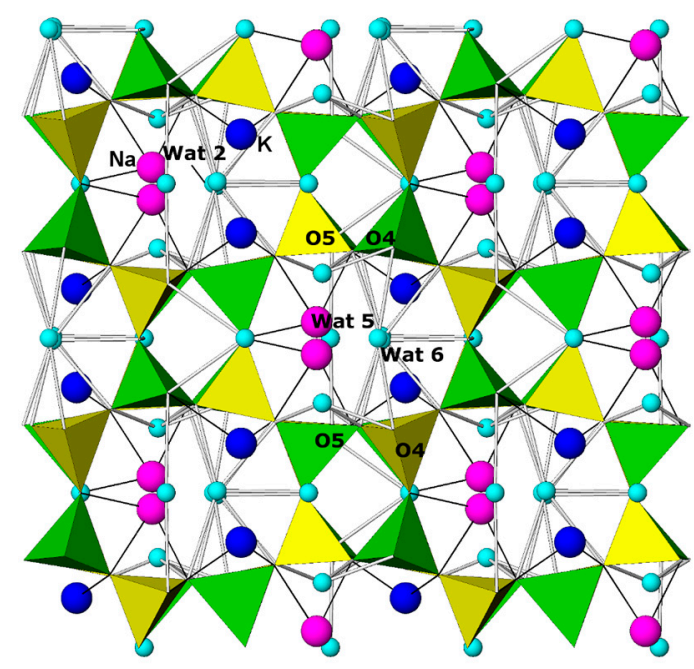

C

Figure 1. Projection of the amicite structure along the [001] direction at (a) $\mathrm{P}_{\mathrm{amb}}$, (b) $1.25 \mathrm{GPa}$, and (c) $4.71 \mathrm{GPa}$. The projection of the structure at $\mathrm{P}_{\mathrm{amb}}(\mathrm{rev})$ is not reported, this being virtually identical to that at $\mathrm{P}_{\mathrm{amb}}$. Purple spheres $=\mathrm{Na}$; blue spheres $=\mathrm{K}$; light blue spheres = water; yellow tetrahedra $=\mathrm{Si}$; green tetrahedra $=\mathrm{Al}$. 


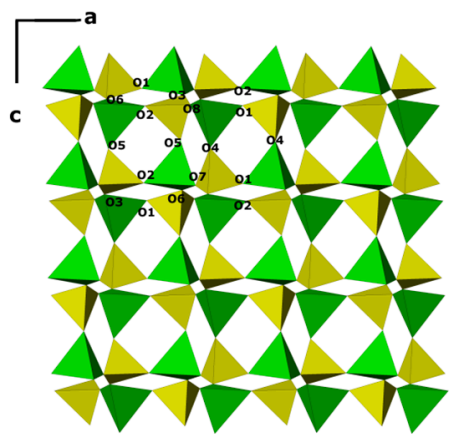

a

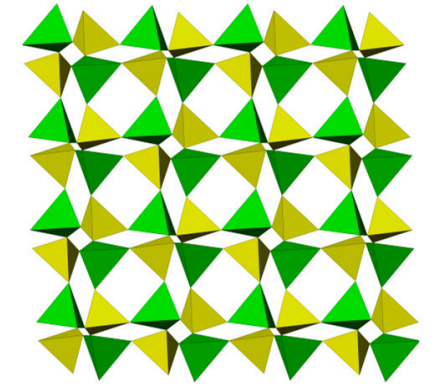

b

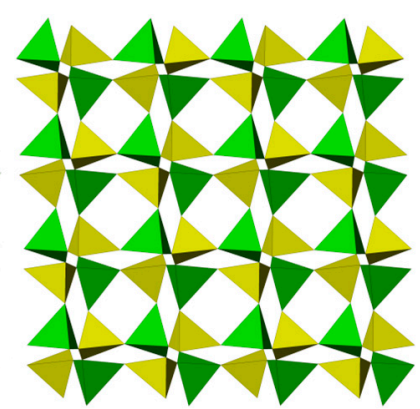

C

Figure 2. Projection of the amicite structure along the [010] direction at (a) $\mathrm{P}_{\mathrm{amb}}$, (b) $1.25 \mathrm{GPa}$, and (c) $4.71 \mathrm{GPa}$. The projection of the structure at $\mathrm{P}_{\mathrm{amb}}(\mathrm{rev})$ is not reported, this being virtually identical to that at $\mathrm{P}_{\mathrm{amb}}$.

\section{Experimental Methods}

In-situ HP XRPD experiments were performed at the SNBL1 (BM01a) beamline at ESRF, using an ETHZ modified Merril-Basset diamond anvil cell (DAC) [29] with flat culets of $600 \mu \mathrm{m}$ in diameter. Powders were loaded into a pre-indented gasket hole (i.e., a stainless steel foil of $60-80 \mu \mathrm{m}$ thickness) with $250 \mu \mathrm{m}$ diameter. The experiments were performed using two different PTM: m.e.w. as nominally penetrating, and s.o. as non-penetrating media, respectively. Pressure was measured before and after data collection at each pressure using the ruby fluorescence method [30] on the non-linear hydrostatic pressure scale [31]. The diffraction data were collected at a wavelength of $0.6825 \AA$ in the Debye-Scherrer geometry on an area detector. One-dimensional diffraction patterns were obtained by integrating the two dimensional images with the program FIT2D [32].

Amicite was compressed up to 8.13(5) GPa in m.e.w. and 8.68(5) GPa in s.o. In the latter case a partial loss of the hydrostatic conditions above $2.8 \mathrm{GPa}$ was observed. In both experiments about 20 images were collected at increasing pressure values. Moreover, some patterns (labeled (rev) in Tables and Figures) were collected upon decompression down to ambient conditions. Figure $3 a, b$ reports selected integrated patterns obtained in m.e.w. and s.o., respectively.

The structural refinements of the data collected in m.e.w. converged successfully up to 4.71(5) GPa. At higher pressure (up to $6.9 \mathrm{GPa}$ ) the refinements were still possible, but some framework bond distances and angles produced unreliable values. As a consequence, above 4.71(5) GPa, only the unit-cell parameters were refined by the Rietveld method in the $2^{\circ}-40^{\circ} 2 \theta$ range.

For amicite in s.o., the low data quality did not allow complete structural refinements. The cell parameters were refined successfully up to 5.48(5) GPa, notwithstanding the previously cited hydrostaticity loss observed above $2.8 \mathrm{GPa}$.

Rietveld profile fitting was performed using the GSAS package [33] with the EXPGUI [34] interface. The initial structural model is as reported in [28]. The background curve was fitted by a Chebyshev polynomial with 20 coefficients. The pseudo-Voigt profile function proposed by [35] was applied, and the peak intensity cut-off was set to $0.1 \%$ of the peak maximum. Soft-restraints were applied to the $\mathrm{T}-\mathrm{O}$ distances $[\mathrm{Si}-\mathrm{O}=1.58(2)-1.62(2) ; \mathrm{Al}-\mathrm{O}=1.72(2)-1.74(2)]$ and their weights were gradually decreased after the initial stages of refinement (up to $F=1$ in GSAS terminology). The isotropic displacement parameters were constrained in the following way: the same value for all the tetrahedral cations, a second value for all the framework oxygen atoms, a third value for the extraframework cations, and a fourth value for the water molecule oxygen atoms. The unit-cell parameters were allowed to vary in all the refinement cycles. Details of the structural refinements are reported in Table 1. 

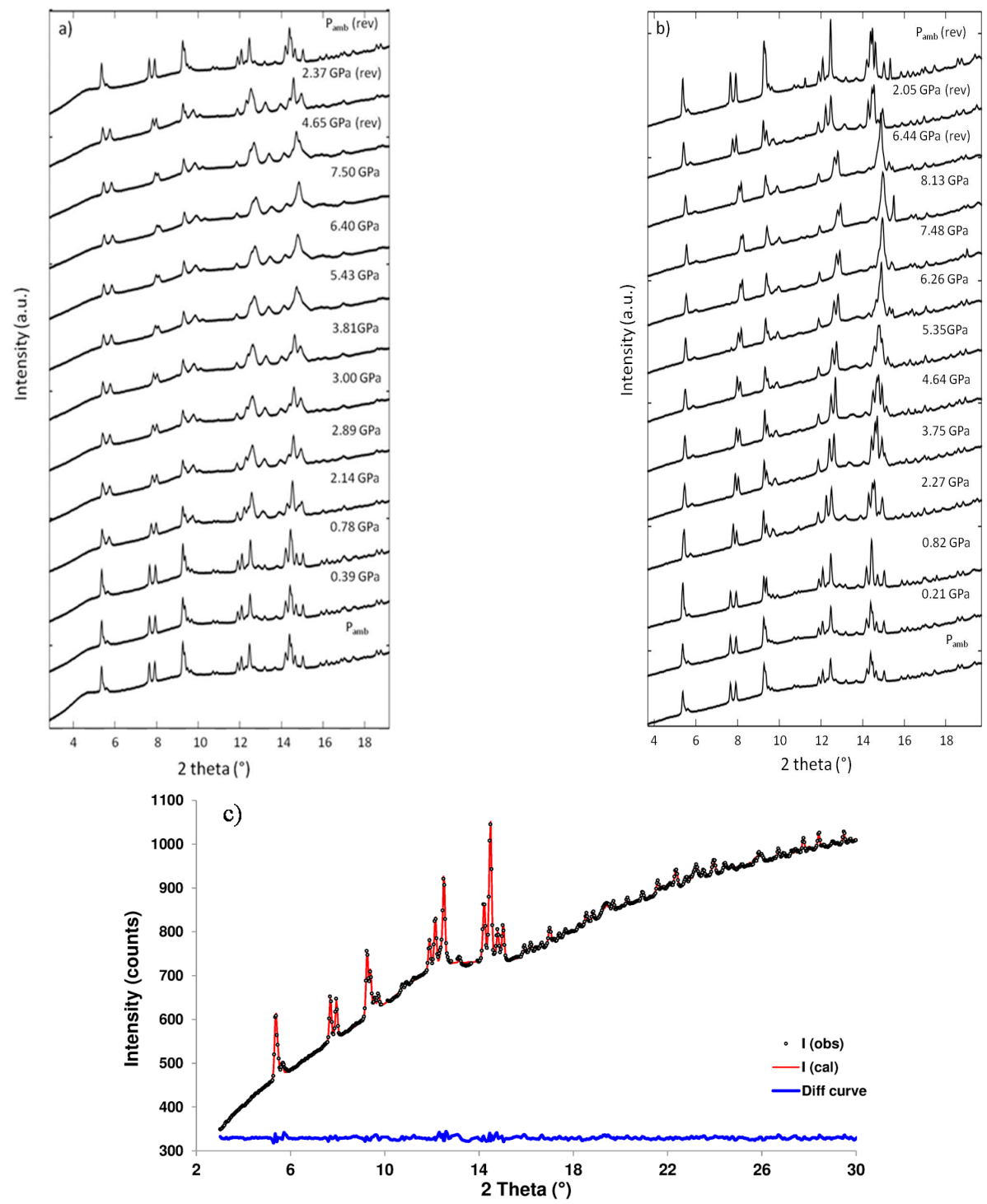

Figure 3. Selected integrated powder patterns, collected in silicone oil (s.o.) (a), and (16:3:1) methanol-ethanol-water (m.e.w.) (b), reported as a function of pressure. The patterns at the top of the figures were collected during decompression. (c) Observed and calculated profiles of the X-ray powder diffraction (XRPD) amicite pattern at 1.25 GPa.

Table 1. Experimental and structural refinement parameters for the $X$-ray powder diffraction (XRPD) measurements performed on amicite in (16:3:1) methanol:ethanol:water (m.e.w.) at $\mathrm{P}_{\mathrm{amb}}, 1.25$ GP, 4.71 GPa, and upon decompression $\left(\mathrm{P}_{\mathrm{amb}}(\mathrm{rev})\right)$.

\begin{tabular}{ccccc}
\hline $\mathbf{P}(\mathbf{G P a})$ & $\mathbf{P}_{\mathbf{a m b}}$ & $\mathbf{1 . 2 5} \mathbf{G P a}$ & $\mathbf{4 . 7 1 ~ G P a}$ & $\mathbf{P}_{\mathbf{a m b}}(\mathbf{r e v})$ \\
\hline Space Group & $I 2$ & $I 2$ & $I 2$ & $I 2$ \\
$a(\AA)$ & $10.2324(8)$ & $10.1882(9)$ & $9.8661(5)$ & $10.2296(5)$ \\
$b(\AA)$ & $10.43456(8)$ & $10.443(1)$ & $10.4966(7)$ & $10.4328(6)$ \\
$c(\AA)$ & $9.8987(7)$ & $9.8651(8)$ & $9.6887(5)$ & $9.8931(4)$ \\
$V\left(\AA^{3}\right)$ & $1056.63(2)$ & $1048.7(2)$ & $1002.59(8)$ & $1055.39(8)$ \\
$\beta\left(^{\circ}\right)$ & $88.382(6)$ & $87.49(9)$ & $87.728(8)$ & $88.349(6)$ \\
$x R_{\mathrm{p}}(\%)$ & 0.7 & 0.7 & 0.7 & 1.0 \\
$R_{\mathrm{wp}}(\%)$ & 0.5 & 0.4 & 0.4 & 1.0 \\
$R \mathrm{~F}^{2}(\%)$ & 11.0 & 15.3 & 16.7 & 11.8 \\
No. of variables & 88 & 94 & 96 & 88 \\
\hline
\end{tabular}




\section{Results and Discussion}

From an inspection of the powder patterns in Figure 3 it is evident that the peak intensities generally decrease and the peak profiles become broader with increasing pressure. These effects could be due to several factors, such as an increase of long-range structural disorder, the presence of texture effects, and in the case of s.o. above $2.8 \mathrm{GPa}$, a decrease in the hydrostaticity of the PTM. However, HP XRPD data demonstrate that amicite does not undergo complete amorphization up to the highest investigated pressure, and the features characteristic of the pattern collected at ambient conditions are almost completely recovered upon decompression in both experiments.

\subsection{Amicite Compressed in Methanol:Ethanol:Water}

From $\mathrm{P}_{\mathrm{amb}}$ to $8.13 \mathrm{GPa}$, the unit-cell volume reduces by about $9.3 \%$, with the unit-cell axes showing a strongly anisotropic behavior $(\Delta a=-6.0 \%, \Delta b=+0.6 \%, \Delta c=-4.1 \%, \Delta \beta=-0.2 \%)$ (Table 2 and Figure 4). In particular, the pseudo-tetragonal $a$ and $c$ axes shrink, while the $b$ axis, which is perpendicular to the dense layers, slightly increases. Cell deformation starts above $0.62 \mathrm{GPa}$ and at P $>3.80 \mathrm{GPa}$ a slight increase in compressibility is observed (Figure $4 \mathrm{~d}$ ).

Table 2. Unit-cell parameters of natural amicite at the investigated pressures, using (16:3:1) methanol:ethanol:water (m.e.w.) and silicon oil (s.o.) as pressure-transmitting media (PTM).

\begin{tabular}{|c|c|c|c|c|c|}
\hline$P(G P a)$ & $a(\AA)$ & $b(\AA)$ & $c(\AA)$ & $V\left(\AA^{3}\right)$ & $\beta\left(^{\circ}\right)$ \\
\hline \multicolumn{6}{|c|}{ amicite (m.e.w.) } \\
\hline$P_{a m b}$ & $10.2324(8)$ & $10.43456(8)$ & $9.8987(7)$ & $1056.63(2)$ & $88.382(6)$ \\
\hline 0.04 & $10.2375(6)$ & $10.4383(6)$ & $9.8949(5)$ & $1056.96(1)$ & $88.343(5)$ \\
\hline 0.09 & $10.2378(7)$ & $10.44088(7)$ & $9.8925(6)$ & $1056.96(2)$ & $88.294(5)$ \\
\hline 0.21 & $10.241(8)$ & $10.4429(7)$ & $9.886(6)$ & 1056.81 & $88.170(5)$ \\
\hline 0.36 & $10.244(6)$ & $10.44(1)$ & $9.8846(8)$ & $1057(1)$ & $88.03(7)$ \\
\hline 0.62 & $10.2447(7)$ & $10.4365(7)$ & $9.8859(6)$ & $1056.4(2)$ & $87.891(6)$ \\
\hline 0.82 & $10.2334(7)$ & $10.4289(8)$ & $9.8857(6)$ & 1054.3(2) & $87.786(7)$ \\
\hline 1.25 & $10.1882(9)$ & $10.443(1)$ & $9.8651(8)$ & $1048.7(2)$ & $87.49(9)$ \\
\hline 1.67 & $10.1290(9)$ & 10.471(1) & $9.8622(8)$ & 1044.7(2) & $87.236(7)$ \\
\hline 2.22 & 10.073(1) & $10.485(1)$ & $9.853(1)$ & $1039.6(3)$ & 87.395(9) \\
\hline 2.75 & $10.027(1)$ & $10.498(1)$ & $9.823(1)$ & 1033.1(3) & $87.52(1)$ \\
\hline 3.23 & $9.9823(6)$ & 10.4975 & $9.7881(5)$ & $1024.78(9)$ & $87.588(9)$ \\
\hline 3.80 & $9.9382(5)$ & $10.5004(6)$ & $9.7507(5)$ & $1016.66(8)$ & 87.633(8) \\
\hline 4.71 & $9.8661(5)$ & $10.4966(7)$ & $9.6887(5)$ & $1002.59(8)$ & $87.728(8)$ \\
\hline 5.35 & $9.820(3)$ & $10.500(4)$ & $9.648(3)$ & 994.1(9) & $87.86(3)$ \\
\hline 6.29 & $9.747(4)$ & $10.503(5)$ & $9.588(4)$ & 981(1) & 87.95(3) \\
\hline 6.71 & $9.717(4)$ & $10.505(5)$ & $9.568(4)$ & $976(1)$ & $88.08(4)$ \\
\hline 6.91 & $9.693(5)$ & $10.502(5)$ & $9.551(4)$ & 972(1) & $88.18(4)$ \\
\hline 7.48 & $9.657(5)$ & $10.500(6)$ & $9.526(5)$ & $966(1)$ & $88.24(5)$ \\
\hline 8.13 & $9.618(6)$ & $10.498(7)$ & $9.496(6)$ & $958(1)$ & $88.24(6)$ \\
\hline 6.45(rev) & $9.732(5)$ & $10.527(5)$ & $9.598(4)$ & $983(1)$ & $88.28(4)$ \\
\hline 4.42(rev) & $9.894(2)$ & $10.528(3)$ & $9.726(2)$ & $1012.4(6)$ & $87.94(2)$ \\
\hline 2.05(rev) & 10.108(1) & $10.481(1)$ & $9.884(1)$ & 1046.1(3) & $87.44(1)$ \\
\hline$P_{\mathrm{amb}}(\mathrm{rev})$ & $10.2296(5)$ & $10.4328(6)$ & $9.8931(4)$ & $1055.39(8)$ & $88.349(6)$ \\
\hline \multicolumn{6}{|c|}{ amicite (s.o.) } \\
\hline $\mathbf{P}_{\mathrm{amb}}$ & $10.2372(9)$ & $10.4352(9)$ & $9.892(8)$ & $1056.30(2)$ & $88.269(8)$ \\
\hline 0.39 & $10.2271(6)$ & $10.4319(7)$ & $9.8686(5)$ & $1052.25(8)$ & 88.071(7) \\
\hline 0.78 & $10.2133(8)$ & $10.4279(9)$ & $9.8528(7)$ & 1048.70(1) & 87.97(1) \\
\hline 1.23 & $10.1923(9)$ & $10.434(1)$ & $9.8411(7)$ & 1045.90(1) & $87.890(1)$ \\
\hline 1.72 & $10.160(1)$ & $10.452(2)$ & $9.828(1)$ & $1043(2)$ & $87.8(2)$ \\
\hline 2.18 & 10.111(2) & $10.468(2)$ & $9.808(2)$ & 1037.4(2) & $87.720(3)$ \\
\hline 2.89 & $10.026(3)$ & $10.505(4)$ & $9.779(8)$ & 1029.4(4) & $88.020(6)$ \\
\hline 3.35 & $9.994(4)$ & $10.519(4)$ & $9.77(3)$ & $1026.6(4)$ & $88.16(6)$ \\
\hline 3.86 & $9.9440(4)$ & $10.535(5)$ & $9.747(3)$ & $1020.7(5)$ & $88.38(7)$ \\
\hline 4.27 & $9.911(4)$ & $10.541(5)$ & $9.729(3)$ & 1016.1(5) & $88.55(4)$ \\
\hline 4.87 & $9.874(3)$ & $10.537(3)$ & $9.717(2)$ & $1010.5(5)$ & $88.3(4)$ \\
\hline 5.48 & $9.816(6)$ & $10.555(6)$ & $9.682(5)$ & $1002.6(2)$ & $88.91(9)$ \\
\hline$P_{a m b}(r e v)$ & $10.239(6)$ & $10.432(7)$ & $9.8981(5)$ & $1056.8(2)$ & $88.26(9)$ \\
\hline
\end{tabular}


The results of the structural refinements corresponding to four selected pressure values $\left(\mathrm{P}_{\mathrm{amb}}, 1.25 \mathrm{GPa}, 4.71 \mathrm{GPa}, \mathrm{P}_{\mathrm{amb}}(\mathrm{rev})\right)$ are reported in Tables 3 and 4 and shown in Figures 1 and 2. The structural variations exhibited by the framework in the range $\mathrm{P}_{\mathrm{amb}}-4.71 \mathrm{GPa}$ regard the shape of both the 4-membered rings, forming the double crankshaft chains, and the 8-membered rings defining the channels along the $a$ and $c$ axes. In particular: (i) the ellipticity-i.e., the ratio between the longest and shortest oxygen-oxygen distance within the 8-ring window-of the channel running along the $a$ axis only slightly decreases, passing from 1.36 at $\mathrm{P}_{\mathrm{amb}}$ to 1.32 at $4.71 \mathrm{GPa}$; (ii) the ellipticity of the channel running along the $c$ axis considerably increases, passing from 1.39 to 1.54; (iii) the tilted 4-rings of the double crankshaft chains-one defined by the distances O7-O8 and O4-O5, the other by O3-O6 and $\mathrm{O} 1-\mathrm{O} 2$ - become a square and a rhombus, respectively; (iv) concerning the two flat 4-rings of the double crankshaft chains, the one defined by the distances $\mathrm{O} 4-\mathrm{O} 4$ and $\mathrm{O} 1-\mathrm{O} 1$ becomes more similar to a square, while the other one defined by $\mathrm{O} 5-\mathrm{O} 5$ and $\mathrm{O} 2-\mathrm{O} 2$ remains almost unchanged.
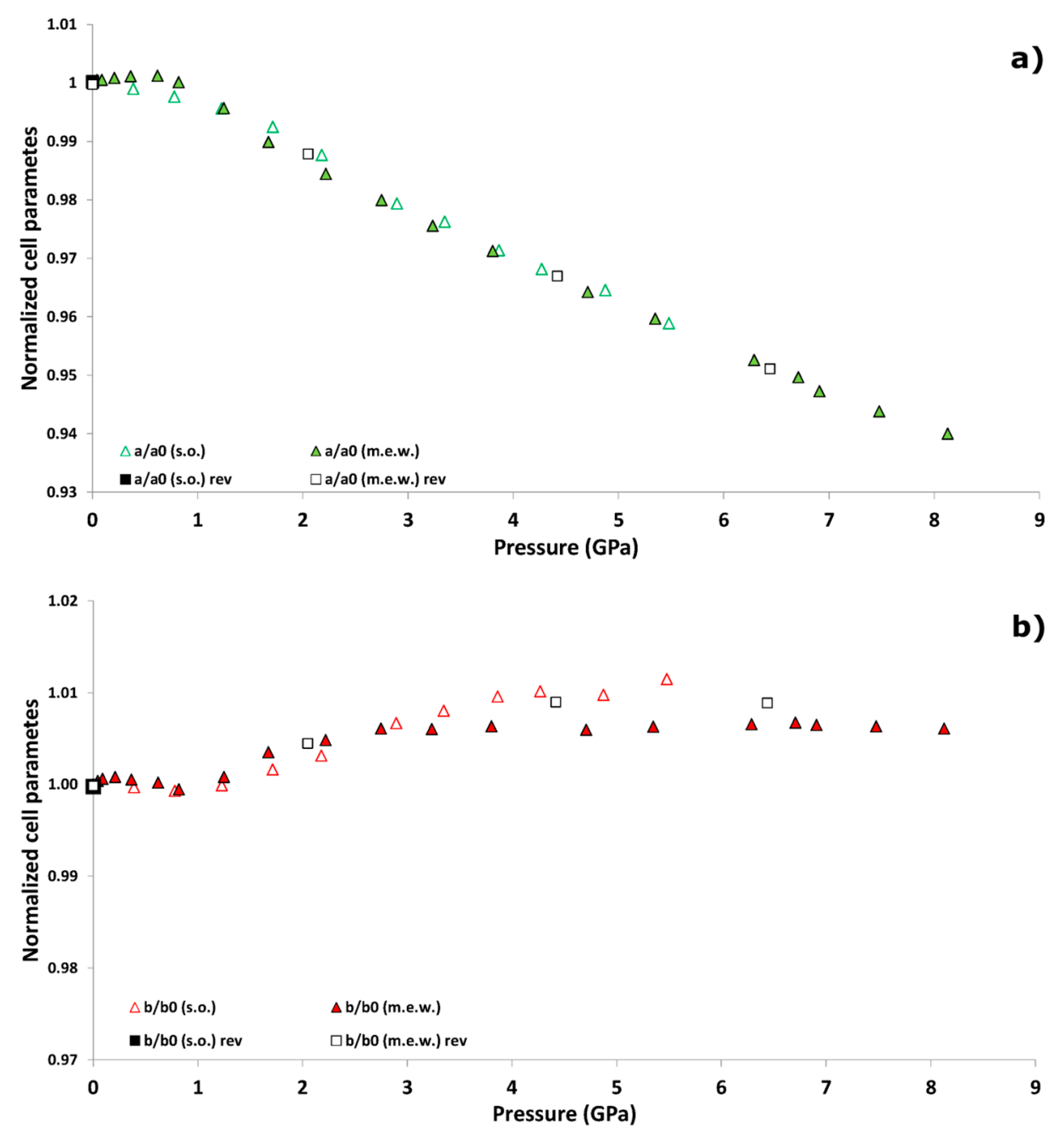

Figure 4. Cont. 

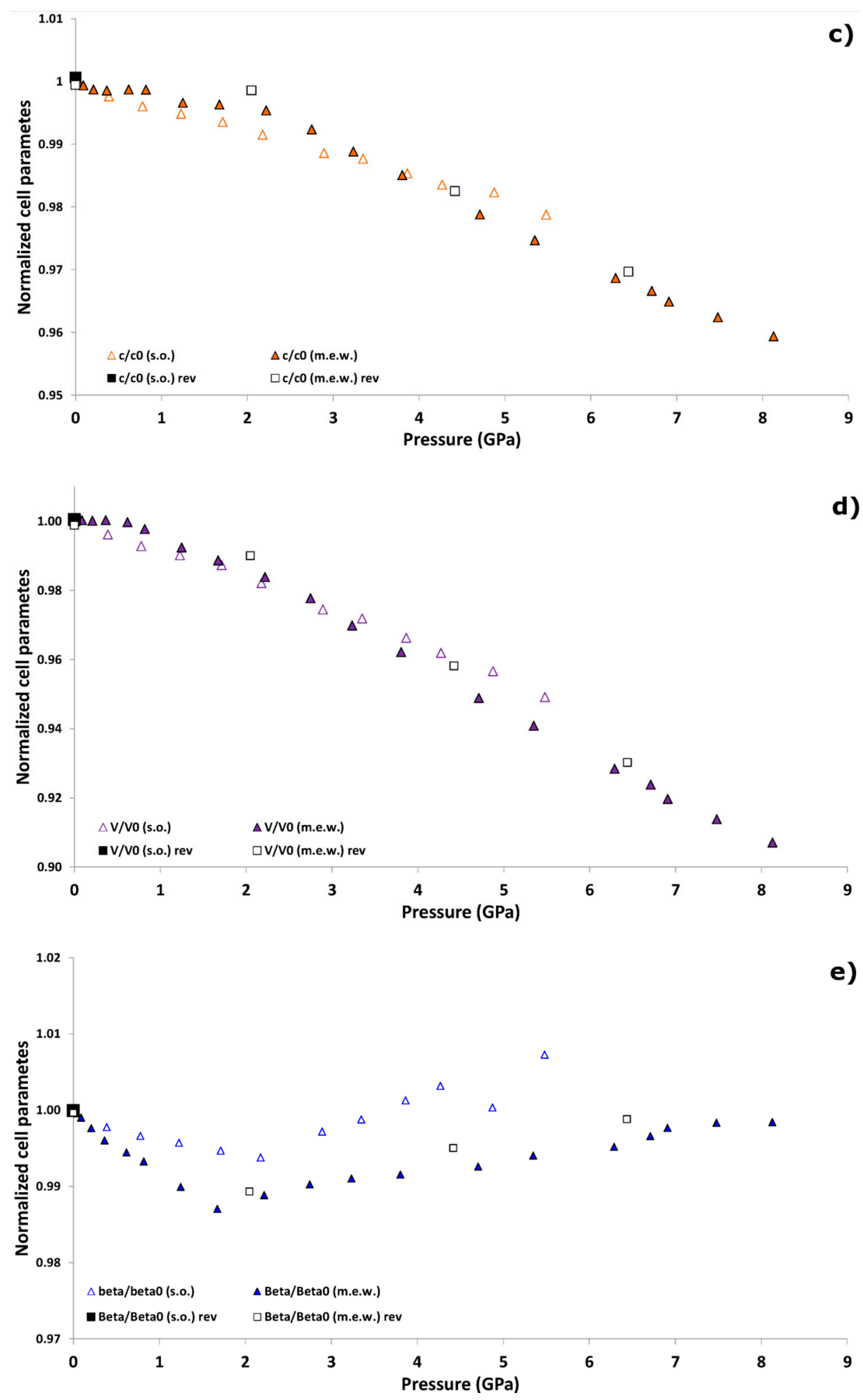

Figure 4. Variation of amicite normalized lattice parameters (a) $a / a 0 ;(\mathbf{b}) b / b 0 ;(\mathbf{c}) c / c 0$; (d) $V / V 0 ;(\mathbf{e}) \beta / \beta 0$ as a function of pressure in silicone oil and (16:3:1) methanol-ethanol-water. The errors associated with the cell parameters are smaller than the symbol size. 
Table 3. Refined atomic positions, occupancy factors, and displacement parameters of amicite at $\mathrm{P}_{\mathrm{amb}}$, 1.25 GPa, 4.71 GPa, and upon decompression $\left(\mathrm{P}_{\mathrm{amb}}(\mathrm{rev})\right)$ in m.e.w.

\begin{tabular}{|c|c|c|c|c|c|}
\hline & $x / a$ & $y / b$ & $\mathrm{z} / \mathrm{c}$ & Occ & Uiso $\left({ }^{*} 100\right)$ \\
\hline & \multicolumn{5}{|c|}{$\mathbf{P}_{\mathrm{amb}}$} \\
\hline $\mathbf{K}$ & $0.324(1)$ & $-0.014(2)$ & $0.960(1)$ & 1 & $0.91(3)$ \\
\hline $\mathrm{Na}$ & $0.438(1)$ & $0.241(2)$ & $0.661(1)$ & 1 & $0.91(3)$ \\
\hline Al1 & $0.153(2)$ & $0.241(3)$ & $0.150(2)$ & 1 & $0.6(3)$ \\
\hline $\mathrm{A} 12$ & $0.152(2)$ & $-0.005(3)$ & $0.658(2)$ & 1 & $0.6(3)$ \\
\hline Si1 & $0.156(2)$ & $-0.023(3)$ & $0.327(2)$ & 1 & $0.6(3)$ \\
\hline $\mathrm{Si} 2$ & $0.159(2)$ & $0.255(3)$ & $0.820(2)$ & 1 & $0.6(3)$ \\
\hline 01 & $0.006(2)$ & $-0.055(4)$ & $0.303(4)$ & 1 & $1.5(3)$ \\
\hline $\mathrm{O} 2$ & $-0.006(2)$ & $0.273(5)$ & $0.205(3)$ & 1 & $1.5(3)$ \\
\hline $\mathrm{O} 3$ & $0.206(3)$ & $0.135(3)$ & $0.730(4)$ & 1 & $1.5(3)$ \\
\hline O4 & $0.176(3)$ & $0.008(5)$ & $0.484(2)$ & 1 & $1.5(3)$ \\
\hline O5 & $0.182(3)$ & $0.226(5)$ & $0.977(2)$ & 1 & $1.5(3)$ \\
\hline O6 & $0.187(4)$ & $0.107(3)$ & $0.247(4)$ & 1 & $1.5(3)$ \\
\hline O7 & $0.255(4)$ & $0.356(3)$ & $0.215(3)$ & 1 & $1.5(3)$ \\
\hline O8 & $0.765(3)$ & $0.386(3)$ & $0.216(4)$ & 1 & $1.5(3)$ \\
\hline Wat1 & $0.307(4)$ & $0.267(6)$ & $0.475(5)$ & $0.75(3)$ & $0.2(6)$ \\
\hline Wat2 & $0.456(3)$ & $0.090(3)$ & $0.243(3)$ & 1 & $0.2(6)$ \\
\hline Wat3 & 0 & $0.300(6)$ & 0.5 & 1 & $0.2(6)$ \\
\hline \multirow[t]{2}{*}{ Wat4 } & 0.5 & $0.42(4)$ & 0.5 & $0.12(3)$ & $0.2(6)$ \\
\hline & \multicolumn{5}{|c|}{$1.25 \mathrm{GPa}$} \\
\hline K & $0.331(1)$ & $-0.001(2)$ & $0.964(1)$ & 1 & $4.5(4)$ \\
\hline $\mathrm{Na}$ & $0.449(2)$ & $0.276(3)$ & $0.672(2)$ & 1 & $4.5(4)$ \\
\hline Al1 & $0.143(1)$ & $0.249(2)$ & $0.147(2)$ & 1 & $0.4(2)$ \\
\hline $\mathrm{Al} 2$ & $0.164(2)$ & $0.007(2)$ & $0.659(2)$ & 1 & $0.4(2)$ \\
\hline Si1 & $0.151(1)$ & $-0.010(2)$ & $0.331(2)$ & 1 & $0.4(2)$ \\
\hline $\mathrm{Si} 2$ & $0.159(1)$ & $0.265(3)$ & $0.822(2)$ & 1 & $0.4(2)$ \\
\hline 01 & $-0.003(1)$ & $-0.031(3)$ & $0.301(3)$ & 1 & $0.7(3)$ \\
\hline $\mathrm{O} 2$ & $-0.008(1)$ & $0.307(3)$ & $0.201(3)$ & 1 & $0.7(3)$ \\
\hline $\mathrm{O} 3$ & $0.218(2)$ & $0.146(3)$ & $0.730(4)$ & 1 & $0.7(3)$ \\
\hline $\mathrm{O} 4$ & $0.182(3)$ & $0.028(4)$ & $0.486(1)$ & 1 & $0.7(3)$ \\
\hline O5 & $0.183(3)$ & $0.230(4)$ & $0.977(2)$ & 1 & $0.7(3)$ \\
\hline O6 & $0.183(3)$ & $0.117(3)$ & $0.241(3)$ & 1 & $0.7(3)$ \\
\hline O7 & $0.256(3)$ & $0.365(3)$ & $0.185(3)$ & 1 & $0.7(3)$ \\
\hline O8 & $0.756(3)$ & $0.389(3)$ & $0.230(2)$ & 1 & $0.7(3)$ \\
\hline Wat1 & $0.283(2)$ & $0.257(5)$ & $0.467(3)$ & 1 & $1.7(5)$ \\
\hline Wat2 & $0.480(3)$ & $0.105(4)$ & $0.277(3)$ & 1 & $1.7(5)$ \\
\hline Wat3 & 0 & $0.259(6)$ & 0.5 & 1 & $1.7(5)$ \\
\hline Wat4 & 0.5 & $0.459(7)$ & 0.5 & $0.74(3)$ & $1.7(5)$ \\
\hline \multirow[t]{2}{*}{ Wat5 } & $0.570(6)$ & $0.172(5)$ & $0.964(4)$ & $0.40(3)$ & $1.7(5)$ \\
\hline & \multicolumn{5}{|c|}{$4.71 \mathrm{GPa}$} \\
\hline $\mathbf{K}$ & $0.340(1)$ & $0.006(2)$ & $0.965(2)$ & 1 & $5.1(5)$ \\
\hline $\mathrm{Na}$ & $0.448(2)$ & $0.276(3)$ & $0.672(2)$ & 1 & $5.1(5)$ \\
\hline Al1 & $0.164(2)$ & $0.259(2)$ & $0.154(2)$ & 1 & $2.1(3)$ \\
\hline $\mathrm{A} 12$ & $0.166(2)$ & $0.024(3)$ & $0.657(2)$ & 1 & $2.1(3)$ \\
\hline Si1 & $0.161(2)$ & $0.009(2)$ & $0.32(2)$ & 1 & $2.1(3)$ \\
\hline $\mathrm{Si} 2$ & $0.152(2)$ & $0.275(3)$ & $0.822(2)$ & 1 & $2.1(3)$ \\
\hline 01 & $0.001(2)$ & $-0.009(4)$ & $0.303(3)$ & 1 & $2.4(4)$ \\
\hline $\mathrm{O} 2$ & $0.004(2)$ & $0.316(4)$ & $0.199(4)$ & 1 & $2.4(4)$ \\
\hline $\mathrm{O} 3$ & $0.220(3)$ & $0.167(3)$ & $0.723(3)$ & 1 & $2.4(4)$ \\
\hline $\mathrm{O} 4$ & $0.206(3)$ & $0.044(4)$ & $0.483(2)$ & 1 & $2.4(4)$ \\
\hline O5 & $0.174(3)$ & $0.229(3)$ & $0.980(2)$ & 1 & $2.4(4)$ \\
\hline O6 & $0.216(3)$ & $0.143(3)$ & $0.265(3)$ & 1 & $2.4(4)$ \\
\hline O7 & $0.225(3)$ & $0.403(3)$ & $0.209(3)$ & 1 & $2.4(4)$ \\
\hline 08 & $0.766(3)$ & $0.404(3)$ & $0.218(4)$ & 1 & $2.4(4)$ \\
\hline Wat1 & $0.290(2)$ & $0.259(5)$ & $0.472(4)$ & 1 & $2.5(5)$ \\
\hline Wat2 & $0.494(3)$ & $0.078(3)$ & $0.247(3)$ & 1 & $2.5(5)$ \\
\hline Wat3 & 0 & $0.301(1)$ & 0.5 & $0.80(3)$ & $2.5(5)$ \\
\hline Wat4 & 0.5 & $0.494(7)$ & 0.5 & 1 & $2.5(5)$ \\
\hline Wat5 & 0.5 & $0.234(7)$ & 0 & 1 & $2.5(5)$ \\
\hline Wat6 & 0 & $0.590(10)$ & 0 & $0.48(3)$ & $2.5(5)$ \\
\hline
\end{tabular}


Table 3. Cont

\begin{tabular}{cccccc}
\hline & $\mathbf{x} / \mathbf{a}$ & $\mathbf{y} / \mathbf{b}$ & $\mathbf{z} / \mathbf{c}$ & Occ & Uiso $\left.{ }^{*} \mathbf{1 0 0}\right)$ \\
\hline \multicolumn{5}{c}{} & \multicolumn{5}{c}{$\mathbf{P}_{\mathbf{a m b}}$ (rev) } \\
\hline K & $0.335(1)$ & $0.002(2)$ & $0.967(1)$ & 1 & $3.0(4)$ \\
Na & $0.446(2)$ & $0.270(3)$ & $0.671(2)$ & 1 & $3.0(4)$ \\
Al1 & $0.143(1)$ & $0.256(2)$ & $0.154(2)$ & 1 & $0.3(2)$ \\
A12 & $0.164(2)$ & $0.017(3)$ & $0.651(2)$ & 1 & $0.3(2)$ \\
Si1 & $0.153(1)$ & $-0.012(2)$ & $0.323(2)$ & 1 & $0.3(2)$ \\
Si2 & $0.166(1)$ & $0.269(2)$ & $0.824(2)$ & 1 & $0.3(2)$ \\
O1 & $-0.004(1)$ & $-0.017(3)$ & $0.303(3)$ & 1 & $0.4(4)$ \\
O2 & $-0.013(1)$ & $0.304(3)$ & $0.198(3)$ & 1 & $0.4(4)$ \\
O3 & $0.220(3)$ & $0.151(3)$ & $0.732(4)$ & 1 & $0.4(4)$ \\
O4 & $0.186(3)$ & $0.027(4)$ & $0.478(2)$ & 1 & $0.4(4)$ \\
O5 & $0.174(4)$ & $0.233(4)$ & $0.983(2)$ & 1 & $0.4(4)$ \\
O6 & $0.183(3)$ & $0.121(3)$ & $0.244(4)$ & 1 & $0.4(4)$ \\
O7 & $0.244(3)$ & $0.374(3)$ & $0.213(3)$ & 1 & $0.4(4)$ \\
O8 & $0.767(3)$ & $0.405(3)$ & $0.218(3)$ & 1 & $0.4(4)$ \\
Wat1 & $0.278(2)$ & $0.271(5)$ & $0.473(3)$ & $0.92(2)$ & $0.2(7)$ \\
Wat2 & $0.449(3)$ & $0.104(2)$ & $0.241(3)$ & 1 & $0.2(7)$ \\
Wat3 & 0 & $0.329(5)$ & 0.5 & 1 & $0.2(7)$ \\
Wat4 & 0.5 & $0.457(3)$ & 0.5 & $0.16(3)$ & $0.2(7)$ \\
\hline
\end{tabular}

Table 4. Framework and extraframework distances (<3.20 $\mathrm{A})$ for amicite at $\mathrm{P}_{\mathrm{amb}}, 1.25 \mathrm{GPa}, 4.71 \mathrm{GPa}$, and upon decompression $\left(\mathrm{P}_{\mathrm{amb}}(\mathrm{rev})\right)$ in m.e.w.

\begin{tabular}{cccccc}
\hline & & $\mathbf{P}_{\text {amb }}$ & $\mathbf{1 . 2 5} \mathbf{G P a}$ & $\mathbf{4 . 7 1 ~ G P a}$ & $\mathbf{P}_{\mathbf{a m b}}$ (rev) \\
\hline A11- & O2 & $1.732(3)$ & $1.721(2)$ & $1.721(3)$ & $1.721(3)$ \\
& O5 & $1.738(3)$ & $1.722(2)$ & $1.721(3)$ & $1.727(3)$ \\
& O6 & $1.732(3)$ & $1.720(2)$ & $1.719(3)$ & $1.720(3)$ \\
A12- & O7 & $1.732(3)$ & $1.720(2)$ & $1.719(3)$ & $1.721(3)$ \\
& O1 & $1.733(3)$ & $1.721(2)$ & $1.720(3)$ & $1.723(3)$ \\
& O3 & $1.731(3)$ & $1.721(2)$ & $1.720(3)$ & $1.721(3)$ \\
& O4 & $1.735(3)$ & $1.721(2)$ & $1.720(3)$ & $1.724(3)$ \\
Si1- & O8 & $1.731(3)$ & $1.720(2)$ & $1.720(3)$ & $1.719(3)$ \\
& O1 & $1.603(3)$ & $1.620(2)$ & $1.620(3)$ & $1.622(3)$ \\
& O4 & $1.606(3)$ & $1.620(2)$ & $1.620(3)$ & $1.624(3)$ \\
& O6 & $1.601(3)$ & $1.620(2)$ & $1.619(3)$ & $1.620(3)$ \\
Si2- & O7 & $1.601(3)$ & $1.620(2)$ & $1.619(3)$ & $1.620(3)$ \\
& O2 & $1.602(3)$ & $1.6202(2)$ & $1.620(3)$ & $1.622(3)$ \\
& O3 & $1.601(3)$ & $1.6203(2)$ & $1.620(3)$ & $1.621(3)$ \\
& O5 & $1.608(3)$ & $1.6211(2)$ & $1.621(3)$ & $1.626(3)$ \\
K- & O8 & $1.600(3)$ & $1.6196(2)$ & $1.620(3)$ & $1.620(3)$ \\
& O3 & $3.07(1)$ & $3.06(2)$ & $3.11(2)$ & $3.06(1)$ \\
& O5 & $2.89(2)$ & $2.78(2)$ & $2.82(1)$ & $2.92(2)$ \\
& O8 & $2.75(1)$ & $2.76(2)$ & $2.77(1)$ & $2.78(1)$ \\
& Wat1 & $2.71(4)$ & $2.86(4)$ & $2.94(1)$ & $2.73(4)$ \\
& Wat2 & $3.19(2)$ & $3.19(3)$ & $2.69(2)$ & $3.16(3)$ \\
& Wat3 & $2.67(4)$ & $3.07(5)$ & $2.69(1)$ & $3.16(2)$ \\
& Wat5 & & $3.20(7)$ & $2.85(1)$ & $2.50(4)$ \\
& Wat5 & & $2.28(8)$ & $2.85(1)$ & \\
Na- & O1 & $2.65(2)$ & $2.38(2)$ & $2.74(1)$ & $2.63(3)$ \\
& O3 & $2.68(1)$ & $2.77(2)$ & $2.52(1)$ & $2.68(2)$ \\
& O8 & $2.822)$ & $2.52(2)$ & $2.68(2)$ & $2.79(2)$ \\
& Wat1 & $2.33(5)$ & $2.70(4)$ & $2.50(2)$ & $2.65(3)$ \\
& Wat1 & $2.92(3)$ & $3.00(2)$ & $2.98(2)$ & $3.13(3)$ \\
& Wat2 & $2.15(3)$ & $2.10(5)$ & $2.29(1)$ & $2.23(3)$ \\
& Wat4 & $2.52(3)$ & $2.59(5)$ & $2.86(1)$ & $2.62(2)$ \\
& Wat5 & & & & \\
& Wat6 & & & $2.54(1)$ & \\
\hline & & & & & \\
& & & & \\
& & & & \\
6
\end{tabular}


Table 4. Cont.

\begin{tabular}{|c|c|c|c|c|c|}
\hline & & $P_{a m b}$ & $1.25 \mathrm{GPa}$ & $4.71 \mathrm{GPa}$ & $P_{a m b}(\mathrm{rev})$ \\
\hline \multirow[t]{11}{*}{ Wat1- } & $\mathbf{K}$ & & $2.86(4)$ & $2.94(1)$ & $2.73(4)$ \\
\hline & $\mathrm{Na}$ & 2.33(5) & $2.70(4)$ & $2.50(2)$ & 2.65(3) \\
\hline & $\mathrm{Na}$ & 2.92(3) & $3.00(2)$ & $2.98(2)$ & $3.13(3)$ \\
\hline & $\mathrm{O} 3$ & $3.02(4)$ & $2.88(3)$ & 2.63(1) & $2.90(3)$ \\
\hline & $\mathrm{O} 4$ & $3.01(6)$ & $2.61(5)$ & $2.37(1)$ & $2.70(5)$ \\
\hline & O6 & $3.09(6)$ & $2.82(4)$ & $2.48(1)$ & $2.93(4)$ \\
\hline & O7 & $2.79(5)$ & $3.03(3)$ & $3.05(1)$ & $2.82(3)$ \\
\hline & Wat2 & & 3.12(5) & & \\
\hline & Wat3 & $3.16(4)$ & $2.89(2)$ & $2.84(1)$ & 2.91(2) \\
\hline & Wat4 & $2.55(3)$ & $3.08(6)$ & & $3.01(2)$ \\
\hline & Wat6 & & & $2.75(1)$ & \\
\hline \multirow[t]{11}{*}{ Wat2- } & K & $3.191(2)$ & $3.19(3)$ & $2.69(2)$ & $3.16(3)$ \\
\hline & K & & & & $3.16(2)$ \\
\hline & $\mathrm{Na}$ & 2.152(3) & $2.10(5)$ & $2.29(1)$ & $2.23(3)$ \\
\hline & $\mathrm{O} 2$ & & $3.13(4)$ & $2.78(2)$ & \\
\hline & $\mathrm{O} 3$ & & $3.11(4)$ & $3.02(1)$ & \\
\hline & O6 & $2.752(3)$ & $3.07(4)$ & $2.77(1)$ & $2.73(3)$ \\
\hline & O7 & & & 2.83(1) & $3.14(2)$ \\
\hline & O8 & & & $3.081)$ & \\
\hline & Wat1 & & 3.12(4) & & \\
\hline & Wat5 & & $2.556(3)$ & 2.911) & \\
\hline & Wat6 & & & $2.42(1)$ & \\
\hline \multirow[t]{4}{*}{ Wat3- } & $K(x 2)$ & $2.67(4)$ & $3.07(5)$ & 2.69(1) & $2.50(4)$ \\
\hline & $\mathrm{O} 2(\mathrm{x} 2)$ & $2.939(6)$ & $3.054(9)$ & 2.911) & $3.009(4)$ \\
\hline & $\begin{array}{l}\mathrm{O} 4 \\
(\mathrm{x} 2)\end{array}$ & & $3.02(5)$ & & \\
\hline & Wat1(x2) & $3.16(4)$ & $2.89(2)$ & $2.84(1)$ & $2.911(2)$ \\
\hline \multirow[t]{3}{*}{ Wat4- } & $\mathrm{Na}(\mathrm{x} 2)$ & $2.52(3)$ & 2.59(5) & $2.86(1)$ & 2.62(2) \\
\hline & O1(x2) & $3.01(4)$ & $2.973(7)$ & 2.93(1) & $3.008(3)$ \\
\hline & Wa & 1 (x2) & $3.08(6)$ & 2.96(1) & $3.01(2)$ \\
\hline \multirow[t]{8}{*}{ Wat5- } & K & & $3.03(6)$ & 2.85(1) & \\
\hline & $\mathbf{K}$ & & $2.19(8)$ & $2.85(1)$ & \\
\hline & $\mathrm{Na}$ & & $3.03(8)$ & & \\
\hline & O5 & & $2.67(8)$ & & \\
\hline & O6 & & $3.20(6)$ & & \\
\hline & O7 & & 3.01(7) & & \\
\hline & Wat2 & & $2.556(3)$ & 2.91(1) & \\
\hline & Wat2 & & & 2.91(1) & \\
\hline \multirow[t]{4}{*}{ Wat6- } & $\begin{array}{l}\mathrm{Na} \\
(\mathrm{x} 2)\end{array}$ & & & $2.54(1)$ & \\
\hline & $\begin{array}{l}\mathrm{O} 4 \\
(\mathrm{x} 2)\end{array}$ & & & $2.97(2)$ & \\
\hline & Wa & $1(\mathrm{x} 2)$ & & $2.75(1)$ & \\
\hline & Wa & $2(x 2)$ & & $2.42(1)$ & \\
\hline
\end{tabular}

Beyond these framework deformations, the most remarkable effect induced on amicite by compression in m.e.w. is the penetration of additional water molecules from the aqueous PTM into the pores (Figure 5a,b). Already at $0.04 \mathrm{GPa}$, the W1 site, originally partially occupied, fills up and W4 increases its occupancy factor. At $0.35 \mathrm{GPa}$ a new water site (W5) appears near the two-fold axis parallel to $b$. Its occupancy factor subsequently tends to increase up to the maximum and the water molecule moves to the two-fold axis (Figures 1 and 5a and Table 3). Another water site (W6) appears at 3.2 GPa with an occupancy factor of about 0.5 , which remains unvaried up to $4.71 \mathrm{GPa}$. Both sites are close to the center of the 8-membered channel parallel to [001]. In the investigated P range the total number of water molecules increases from 9.24 to 14.58 (see Table 3 and Figure 5b). The cations and the original water molecules undergo only slight positional changes and the new water sites W5 and W6 enter into the coordination sphere of $\mathrm{K}$ and $\mathrm{Na}$, respectively. 

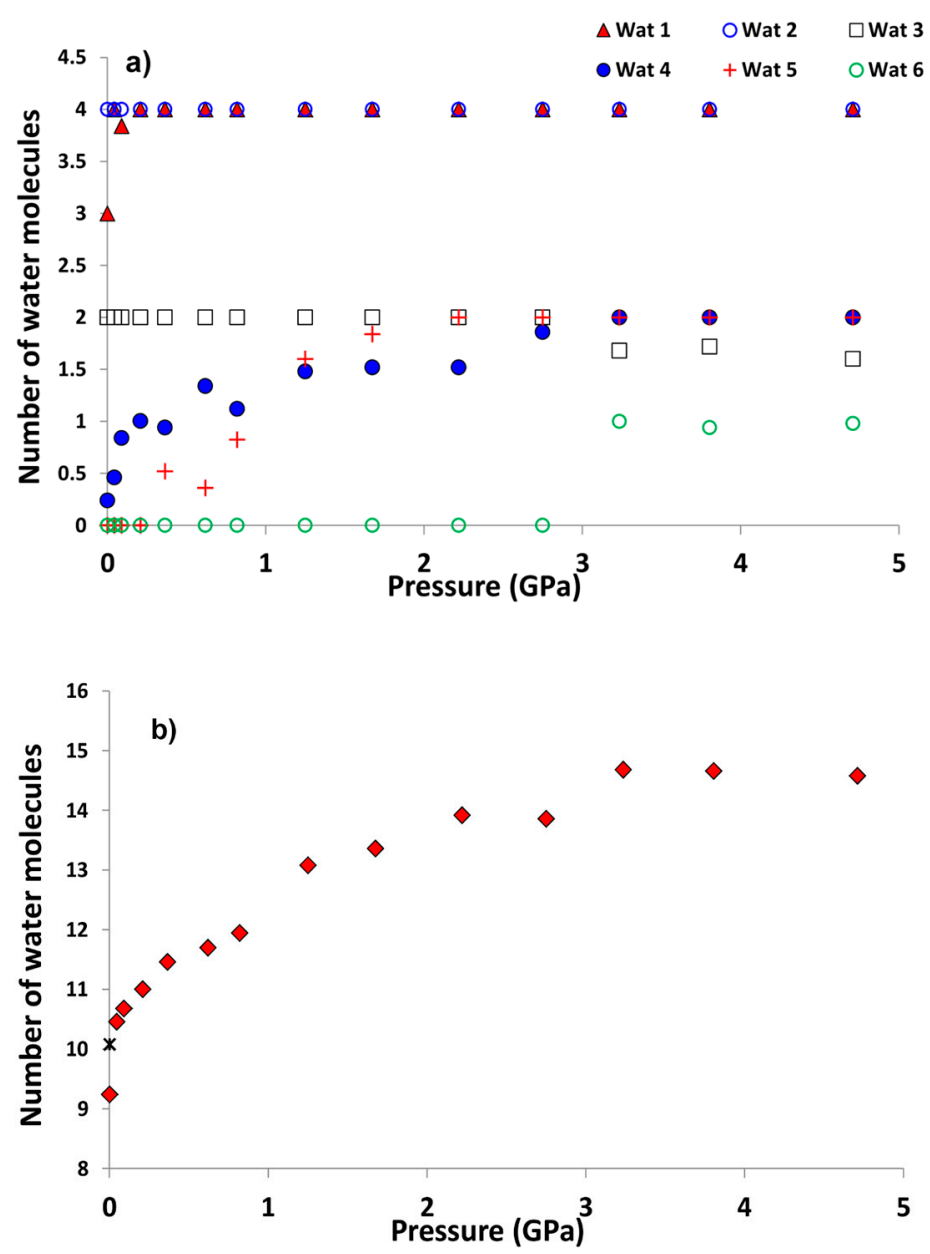

Figure 5. (a) Number of water molecules in the Wat1-Wat6 extraframework sites in amicite compressed in m.e.w. as a function of pressure; (b) total number of water molecules as a function of P. The cross indicates the number of water molecules after pressure release.

In Figure $4 \mathrm{~d}$ two slope changes are visible in the $\mathrm{P}-\mathrm{V}$ diagram at $\mathrm{P}>0.65$ and $\mathrm{P}>3.2 \mathrm{GPa}$, and these can be considered as strictly related to the water penetration. In fact, the rather strong bonds W5-O5 and W6-O4 between the new water sites and the framework, and the decrease in the distances between $\mathrm{W} 1$ and the oxygen atoms O3, O4, O6 (Table 4)—along with the W1 filling-contribute to the $a$ axis contraction, which is the main effect responsible of amicite compression behavior.

The structure refinement performed after $\mathrm{P}$ release to ambient conditions $\left(\mathrm{P}_{\mathrm{amb}}(\mathrm{rev})\right)$, shows that the unit cell parameters (Table 2) and all the structural features (Tables 3 and 4) recover their original values. The W5 and W6 sites disappear and only W1 maintains a higher occupancy factor compared to the original amicite [28]. Overall, less than one additional water molecule of the PTM remains in the structure.

\subsection{Amicite Compressed in Silicone Oil}

Table 2 and Figure 4 show the P-dependence of the lattice parameters of amicite in s.o. It can be seen that the pseudotetragonal $a$ and $c$ axes initially decrease by approximately the same percentage, while the $b$ axis slightly increases. The $a$ and $c$ parameters decrease quite regularly, with a slight slope increase between 2 and $3 \mathrm{GPa}$, particularly evident for the $a$ parameter. In the range $\mathrm{P}_{\mathrm{amb}}-5.43 \mathrm{GPa}$, the overall cell parameter variations are: $\Delta a=-4.1 \%, \Delta b=+1.1 \%, \Delta c=-2.1 \%, \Delta \beta=-0.7 \%$, while the cell volume decreases by approximately $5.1 \%$. Again in s.o. the P-induced effects on the amicite unit cell are reversible upon decompression, as shown in Table 2 and Figure 4. 


\section{Comparison between Amicite Compressibility in Aqueous Medium and Silicone Oil}

The main difference between amicite HP behavior in m.e.w. and s.o. is the higher compressibility in the aqueous medium (see Figure 4 and Table 2). This is clear by comparing the unit cell volume decrease in the two PTM at similar pressure values: $5.1 \%$ at $5.43 \mathrm{GPa}$ in s.o., and $5.9 \%$ at $5.35 \mathrm{GPa}$ in m.e.w. This effect is anomalous compared to what is generally observed for zeolites, when water penetration provides a support against the effects of pressure (e.g., see a review in Table 4 in [36]) [12,13,37,38]. Although detailed structural data for the ramp in s.o. are lacking, this result can be ascribed to formation, during compression in m.e.w., of rather strong bonds between the additional water molecules and the framework oxygen atoms, which contribute to the shrinkage of the $a$ parameter. In particular, the distance O3-O6, which is parallel to the $a$ axis and corresponds to the shortest diameter of the 8-ring perpendicular to $c$, undergoes a $10 \%$ reduction passing from $6.20 \AA$ at $\mathrm{P}_{\mathrm{amb}}$ to $5.58 \AA$ at $4.71 \mathrm{GPa}$ (see Figure 1).

\section{Compressibility Behavior of Microporous Materials with GIS Topology}

A number of microporous materials with GIS topology have been investigated under HP. Among the natural zeolites, amicite, the K-Na member of the GIS family, can be compared to gismondine [11,22], the Ca member, producing the following observations:

1. Compression of gismondine in both m.e.w. and s.o. favors the tetragonalization of the unit cell; in amicite the $a$ and $c$ axes also tend to become more similar at HP, but the beta angle does not substantially change;

2. Gismondine compressed in m.e.w. undergoes a transition to a triclinic phase at about $3 \mathrm{GPa}$; the original symmetry of amicite, by contrast, is maintained in both the experiments;

3. The HP framework deformation mechanism is the same in the two zeolites, essentially being driven by the distortion of the "double crankshaft" chains and the consequent change in the 8-ring channel shape;

4. Amicite's compressibility increases at HP both in m.e.w. and s.o.; by contrast, gismondine's compressibility in s.o. slightly decreases while in m.e.w. it remains constant;

5. PIH occurs in both amicite and in gismondine compressed in m.e.w. However, it induces different reorganizations in the water molecule systems: in amicite there is both the filling of partially occupied sites and the appearance of two new water sites; in gismondine four partially occupied water sites reduce to only two fully occupied sites, giving rise to a more ordered water system;

6. In amicite 5.34 water molecules enter the zeolite porosities when compressed in m.e.w., while in gismondine only one additional molecule penetrates. This result can be explained by the higher channel stuffing of gismondine at $\mathrm{P}_{\mathrm{amb}}$ compared to amicite;

7. Both amicite and gismondine are more compressible in m.e.w. than in s.o., but for different reasons. In gismondine this effect has been justified by the re-organization of the water molecule system, which leaves a larger free volume inside the pores compared to the phase compressed in s.o. In amicite the higher compressibility at HP results from the strong bonds between framework oxygen atoms and the new water molecules;

8. Overall, gismondine is more compressible than amicite, both in m.e.w. and in s.o. Comparing the unit cell volume decrease of the two phases at a similar pressure value-about $5.5 \mathrm{GPa}$-we find $\Delta V=-7.5 \%$ and $-6.4 \%$ for gismondine in m.e.w. and s.o., respectively, while for amicite these values are $-5.9 \%$ and $-5.1 \%$, respectively. The presence of the large potassium cations and the higher number of extraframework sites after PIH in amicite compared to gismondine probably contribute to better supporting the amicite structure. 
Lee and co-workers [23] studied the compressibility in m.e.w. of the potassium gallo silicate K-GaSi-GIS with GIS framework type (ideal formula $\mathrm{K}_{5.76} \mathrm{Ga}_{5.76} \mathrm{Si}_{10.24} \mathrm{O}_{32} \cdot 9.9 \mathrm{H}_{2} \mathrm{O}$, s.g. $I_{1} / a$ ). When the results of this study are compared with those obtained from the natural phases, the following observations can be made:

(a) The main feature of the P-induced evolution of cell parameters of K-GaSi-GIS is the noticeable squashing of the $c$ axis, which is perpendicular to the dense plane and corresponds to the $b$ axis of gismondine and amicite. This response to hydrostatic pressure corresponds to a gradual flattening of the double crankshaft chains and a reduction in the ellipticity of the 8-ring windows. The different behavior compared to amicite and gismondine, where the $b$ axis slightly increases or remains almost unvaried, could be explained by the lower channel stuffing of the K-GaSi-GIS phase related to the high $\mathrm{Si} / \mathrm{Ga}$ ratio;

(b) In K-GaSi-GIS a PIH effect is again observed, with the penetration of about two water molecules at $\mathrm{P}<1 \mathrm{GPa}$, but in this case the overhydration induces a disordering of the K-water system along the channels.

The potassium alumino germanate K-AlGe-GIS with GIS topology (ideal formula $\mathrm{K}_{8} \mathrm{Al}_{8} \mathrm{Ge}_{8} \mathrm{O}_{32} \cdot 8 \mathrm{H}_{2} \mathrm{O}$, s.g. I2/a) was studied under HP by Jang et al. [24]. Its structure is similar to amicite for the ordered distribution of the tetrahedral cations and the same number of extraframework cations. However, there are eight instead of 10 water molecules in the synthetic phase. The variation in the unit cell parameters was determined in m.e.w. up to $3.22 \mathrm{GPa}$, but no structural refinements were reported, so no hypotheses were made concerning a possible PIH. The compressibility is anisotropic with a decrease in the $a$ and $c$ axes, parallel to the channels, of $1.3 \%$ and $1.0 \%$, respectively, while the $b$ parameter, perpendicular to the channels and the double crankshaft chains, decreased by $5.4 \%$ resulting in an almost linear volume contraction of $7.5 \%$. The large $b$ variation is strictly related to a flattening of the double crankshaft chains under P, as already observed for K-GaSi-GIS. Again in this case, the different compressibility behavior compared to amicite and gismondine can be explained by the lower water content and consequent channel stuffing.

\section{Conclusions}

The high-pressure behavior of amicite, a GIS framework type zeolite, was investigated and the strong influence on compressibility of the chemical composition of both the framework and extraframework species was also confirmed for this variety. In particular, the study confirms that the compressibility of microporous materials is not simply related to their framework density and topology, but is also greatly affected by the type, amount, and location of the extra-framework species.

The HP framework deformation mechanism is the same in all the phases with GIS topology and is essentially driven by the distortion of the "double crankshaft" chains and the consequent shape change of the 8-ring channels. However, the degree of compressibility varies due to the different chemical compositions. In these zeolites the pressure-induced penetration of water molecules does not induce a unit cell volume expansion, and in the natural phases, when the structure after P release was determined, the overhydration effects are reversible following the return to ambient conditions.

Acknowledgments: This work was supported by the Italian MIUR, within the frame of the following projects: PRIN2015 "ZAPPING" High-pressure nano-confinement in Zeolites: the Mineral Science know-how APPlied to engineerING of innovative materials for technological and environmental applications" (2015HK93L7), and FIRB, Futuro in Ricerca "Impose Pressure and Change Technology" (RBFR12CLQD). Vladimir Dmitriev and the ESRF BM01 beamline staff are acknowledged for their technical support during the high-pressure XRPD experiments.

Author Contributions: Rossella Arletti, Simona Quartieri, and Giovanna Vezzalini conceived and designed the experiments; Rossella Arletti, Carlotta Giacobbe, and Giovanna Vezzalini performed the experiments; Rossella Arletti and Carlotta Giacobbe analyzed the data; Rossella Arletti, Simona Quartieri, and Giovanna Vezzalini wrote the paper.

Conflicts of Interest: The authors declare no conflict of interest. 


\section{References}

1. Gillet, P.; Malezieux, J.M.; Itie, J.P. Phase changes and amorphization of zeolites at high pressure: The case of scolecite and mesolite. Am. Mineral. 1996, 81, 651-657. [CrossRef]

2. Huang, Y.; Havenga, E.A. Why do zeolites with LTA structure undergo reversible amorphization under pressure? Chem. Phys. Lett. 2001, 345, 65-71. [CrossRef]

3. Rutter, M.D.; Uchida, T.; Secco, R.A.; Huang, Y.; Wang, Y. Investigation of pressure-induced amorphization in hydrated zeolite Li-A and Na-A using synchrotron X-ray diffraction. J. Phys. Chem. Solids 2001, 62, 599-606. [CrossRef]

4. Greaves, G.N.; Meneau, F.; Sapelkin, A.; Colyer, L.M.; Gwynn, I.A.; Wade, S.; Sankar, G. The rheology of collapsing zeolites amorphized by temperature and pressure. Nat. Mater. 2003, 2, 622-629. [CrossRef] [PubMed]

5. Gulìn-González, J.; Suffritti, G.B. Amorphization of calcined LTA zeolites at high pressure: A computational study. Microporous Mesoporous Mater. 2004, 69, 127-134. [CrossRef]

6. Goryainov, S.V. Pressure-induced amorphization of $\mathrm{Na}_{2} \mathrm{Al}_{2} \mathrm{Si}_{3} \mathrm{O}_{10} \cdot 2 \mathrm{H}_{2} \mathrm{O}$ and $\mathrm{KAlSi}_{2} \mathrm{O}_{6}$ zeolites. Phys. Status Solidi 2005, 202, R25-R27. [CrossRef]

7. Secco, R.A.; Huang, Y. Pressure-induced disorder in hydrated Na-A zeolite. J. Phys. Chem. Solids 1999, 60, 999-1002. [CrossRef]

8. Rutter, M.D.; Secco, R.A.; Huang, Y. Ionic conduction in hydrated zeolite Li-, Na- and K-A at high pressures. Chem. Phys. Letter 2000, 331, 189-195. [CrossRef]

9. Lee, Y.; Vogt, T.; Hriljac, J.A.; Parise, J.B.; Artioli, G. Pressure-induced volume expansion of zeolites in the natrolite family. J. Am. Chem. Soc. 2002, 124, 5466-5475. [CrossRef] [PubMed]

10. Lee, Y.; Hriljac, J.A.; Vogt, T. Pressure-induced migration of zeolitic water in laumontite. Phys. Chem. Miner. 2004, 31, 421-428. [CrossRef]

11. Ori, S.; Quartieri, S.; Vezzalini, G.; Dmitriev, V. Pressure-induced over-hydration and water ordering in gismondine: A synchrotron powder diffraction study. Am. Mineral. 2008, 93, 1393-1403. [CrossRef]

12. Arletti, R.; Quartieri, S.; Vezzalini, G. Elastic behavior of zeolite boggsite in silicone oil and aqueous medium: A case of high-pressure-induced over-hydration. Am. Mineral. 2010, 95, 1247-1256. [CrossRef]

13. Quartieri, S.; Montagna, G.; Arletti, R.; Vezzalini, G. Elastic behavior of MFI-type zeolites: Compressibility of H-ZSM-5 in penetrating and non-penetrating media. J. Solid State Chem. 2011, 184, 1505-1516. [CrossRef]

14. Lotti, P.; Gatta, G.D.; Comboni, D.; Merlini, M.; Pastero, L.; Hanfland, M. AlPO4-5 zeolite at high pressure: Crystal-fluid interaction and elastic behavior. Microporous Mesoporous Mater. 2016, 228, 158-167. [CrossRef]

15. Lee, Y.; Hriljac, J.A.; Vogt, T. Pressure-induced argon insertion into an auxetic small pore zeolite. J. Phys. Chem. C 2010, 114, 6922-6927. [CrossRef]

16. Seoung, D.; Lee, Y.; Cynn, H.; Park, C.; Choi, K.Y.; Blom, D.A.; Evans, W.J.; Kao, C.C.; Vogt, T.; Lee, Y. Irreversible xenon insertion into a small-pore zeolite at moderate pressures and temperatures. Nat. Chem. 2014, 6, 835-839. [CrossRef] [PubMed]

17. Lee, Y.; Liu, D.; Seoung, D.; Liu, Z.; Kao, C.C.; Vogt, T. Pressure- and heat-induced insertion of $\mathrm{CO}_{2}$ into an auxetic small-pore zeolite. J. Am. Chem. Soc. 2011, 133, 1674-1677. [CrossRef] [PubMed]

18. Santoro, M.; Scelta, D.; Dziubek, K.; Ceppatelli, M.; Gorelli, F.A.; Bini, R.; Garbarino, G.; Thibaud, J.M.; Di Renzo, F.; Cambon, O.; et al. Synthesis of 1D polymer/zeolite nanocomposites under high pressure. Chem. Mater. 2016, 28, 4065-4071. [CrossRef]

19. Gatta, G.D.; Lee, Y. Zeolites at high pressure: A review. Mineral. Mag. 2014, 78, 267-291. [CrossRef]

20. Baerlocher, C.; McCusker, L.B.; Olson, D.H. Atlas of Zeolite Framework Types, 6th ed.; Elsevier: Amsterdam, The Netherlands, 2007.

21. Vezzalini, G.; Quartieri, S.; Alberti, A. Structural modifications induced by dehydration in the zeolite gismondine. Zeolites 1993, 13, 34-42. [CrossRef]

22. Betti, C.; Fois, E.; Mazzucato, E.; Medici, C.; Quartieri, S.; Tabacchi, G.; Vezzalini, G.; Dmitriev, V. Gismondine under HP: Deformation mechanism and re-organization of the extra-framework species. Microporous Mesoporous Mater. 2007, 103, 190-209. [CrossRef]

23. Lee, Y.; Kim, S.J.; Kao, C.C.; Vogt, T. Pressure-induced hydration and order-disorder transition in a synthetic potassium gallosilicate zeolite with gismondine topology. J. Am. Chem. Soc. 2008, 130, 2842-2850. [CrossRef] [PubMed] 
24. Jang, Y.N.; Kao, C.C.; Vogt, T.; Lee, Y. Anisotropic compression of a synthetic potassium aluminogermanate zeolite with gismondine topology. J. Solid State Chem. 2010, 183, 2305-2308. [CrossRef]

25. Rocha, J.; Lin, Z. Micro and mesoporous mineral phases. Rev. Mineral. Geochem. 2005, 57, 173-201. [CrossRef]

26. Danisi, R.M.; Armbruster, T.; Arletti, R.; Gatta, G.D.; Vezzalini, G.; Quartieri, S.; Dmitriev, V. Elastic behavior and pressure-induced structural modifications of the microporous $\mathrm{Ca}(\mathrm{VO}) \mathrm{Si}_{4} \mathrm{O}_{10} \cdot 4 \mathrm{H}_{2} \mathrm{O}$ dimorphs cavansite and pentagonite. Microporous Mesoporous Mater. 2015, 204, 257-268. [CrossRef]

27. Coombs, D.S.; Alberti, A.; Armbruster, T.; Artioli, G.; Colella, C.; Galli, E.; Grice, J.D.; Liebau, F.; Mandarino, J.A.; Minato, H.; et al. Recommended nomenclature for zeolite minerals: Report of the subcommittee on zeolites of the international mineralogical association, commission on new minerals and minerals names. Can. Mineral. 1997, 35, 1571-1606.

28. Alberti, A.; Vezzalini, G. The crystal structure of amicite, a zeolite. Acta Cryst. 1979, B35, $2866-2869$. [CrossRef]

29. Miletich, R.; Allan, D.R.; Kush, W.F. High-temperature and high-pressure crystal chemistry. Rev. Mineral. Geochem. 2000, 41, 445-519. [CrossRef]

30. Forman, R.A.; Piermarini, G.J.; Barnett, J.D.; Block, S. Pressure measurement made by the utilization of ruby sharp-line luminescence. Science 1972, 176, 4673-4676. [CrossRef] [PubMed]

31. Mao, H.K.; Xu, J.; Bell, P.M. Calibration of the ruby pressure gauge to $800 \mathrm{kbar}$ under quasi-hydrostatic conditions. J. Geophys. Res. 1986, 9, 4673-4676. [CrossRef]

32. Hammersley, A.P.; Svensson, S.O.; Hanfland, M.; Fitch, A.N.; Häusermann, D. Two dimensional detector software: From real detector to idealized image or two-theta scan. High Press. Res. 1996, 14, 235-248. [CrossRef]

33. Larson, A.C.; von Dreele, R.B. GSAS-General Structure Analysis System; Report LAUR 86-748; Los Alamos National Laboratory: Los Alamos, NM, USA, 1996.

34. Toby, B.H. EXPGUI, a graphical user interface for GSAS. J. Appl. Cryst. 2001, 34, 210-213. [CrossRef]

35. Thomson, P.; Cox, D.E.; Hastings, J.B. Rietveld refinement of Debye-Scherrer synchrotron X-ray data from $\mathrm{Al}_{2} \mathrm{O}_{3}$. J. Appl. Cryst. 1987, 20, 79-83. [CrossRef]

36. Colligan, M.; Forster, P.M.; Cheetham, A.K.; Lee, Y.; Vogt, T.; Hriljac, J.A. Synchrotron X-ray powder diffraction and computational investigation of purely siliceous zeolite Y under pressure. J. Am. Chem. Soc. 2004, 126, 12015-12022. [CrossRef] [PubMed]

37. Likhacheva, A.Y.; Seryotkin, Y.V.; Manakov, A.Y.; Goryainov, S.V.; Ancharov, A.I.; Sheromov, M.A. Anomalous compression of scolecite and thomsonite in aqueous medium to 2 GPa. High Press. Res. 2006, 26, 449-453. [CrossRef]

38. Likhacheva, A.Y.; Seryotkin, Y.V.; Manakov, A.Y.; Goryainov, S.V.; Ancharov, A.I.; Sheromov, M.A. Pressure-induced over-hydration of thomsonite: A synchrotron powder diffraction study. Am. Mineral. 2007, 92, 1610-1615. [CrossRef]

(C) 2017 by the authors; licensee MDPI, Basel, Switzerland. This article is an open access article distributed under the terms and conditions of the Creative Commons Attribution (CC BY) license (http:/ / creativecommons.org/licenses/by/4.0/). 